\title{
Anatomical Variations of Guyon's Canal Contents: A Case Report and Incidence in South Australian Population
}

\author{
Mounir N. Ghabriel*
}

Discipline of Anatomy and Pathology, School of Medical Sciences, Faculty of Health Sciences, The University of Adelaide, Frome Road, Adelaide, South Australia 5005, Australia

\begin{abstract}
Ulnar nerve compression has been reported in association with variations in the contents of Guyon's canal, such as the presence of accessory muscles. In the current case study we report on two variations found concurrently in the same hand. Firstly, the presence of an accessory head of the abductor digiti minimi that originated principally from the tendon of palmaris longus in the lower third of the forearm, descended superficial to the ulnar nerve and artery in Guyon's canal, and joined three other heads of the same muscle in the hypothenar eminence. Secondly, the ulnar nerve within the canal showed trifurcation into superficial, deep and muscular branches. A study of 120 hands from cadavers of South Australian population revealed the presence of an accessory head of the abductor digiti minimi that occupied Guyon's canal in $2.5 \%$ of the cases, a much lower incidence than previously reported in other populations. Trifurcation of the ulnar nerve seen here was also noted in the left hand of the same cadaver, but without any accessory muscles in Guyon's canal. Knowledge of such variations is relevant to hand surgery.
\end{abstract}

Keywords: Guyon's canal, entrapment neuropathy, upper limb, accessory head, hand surgery, hypothenar muscles, nerve block.

\section{INTRODUCTION}

The ulnar nerve enters the hand via Guyon's canal between the tendon of flexor carpi ulnaris and the ulnar artery. In clinical practice, the ulnar nerve may be blocked at the distal skin crease of the wrist, lateral to the tendon of flexor carpi ulnaris just proximal to Guyon's canal. The ulnar nerve may also be compressed within the canal by any space-occupying lesion such as lipoma [1], ulnar artery thrombosis [2] or anomalous muscles [3]. Within the canal and usually just distal to the pisiform bone the ulnar nerve divides into a deep branch that supplies short muscles in the hand and a superficial branch which is mainly sensory. Here we report a case with dual variations in Guyon's canal contents: an accessory muscular head of the abductor digiti minimi (ADM) and a distinct ulnar nerve trifurcation. In addition, a systematic study was undertaken to report on the incidence of anatomical variations in the contents of Guyon's canal in South Australian populations.

\section{MATERIALS AND METHODS}

The contents of Guyon's canal in 120 hands were examined for anatomical variations. 98 hands from the anatomical collection in our discipline were studied. In addition 22 hands from 11 cadavers were dissected, and in these the right and left hands were compared. All cadavers were of European descent. The findings were digitally recorded.

*Address correspondence to this author at the Discipline of Anatomy and Pathology, School of Medical Sciences, Faculty of Health Sciences, The University of Adelaide, Frome Road, Adelaide, South Australia 5005, Australia; Tel: +618-83135481; Fax: +618-83134398;

E-mails: mounir.ghabriel@adelaide.edu.au,mounir.ghabriel@gmail.com

\section{RESULTS}

In all dissections Guyon's canal contained the ulnar nerve located lateral to the pisiform bone and the ulnar artery lateral to the ulnar nerve. The ulnar nerve typically divided into superficial and deep branches just distal to the pisiform bone. Three hands however, showed an accessory head of the abductor digiti mini (ADM) located superficial to the neurovascular bundle within the canal and extended proximally into the forearm. One of these hands was the right hand of a cadaver of a 79-year-old male in whom the accessory head of the ADM was of substantial size. The fleshy belly of this accessory head of the ADM muscle, originated from a broad aponeurosis attached along a length of $4 \mathrm{~cm}$ to the medial border of the tendon of palmaris longus, and its most proximal origin was $6 \mathrm{~cm}$ proximal to the flexor retinaculum (Fig. 1). Additional muscle fibres originated from the overlying deep fascia. The fusiform red belly started $4.5 \mathrm{~cm}$ proximal to the pisiform bone, and was flat at its origin, but assumed a cylindrical form, being one $\mathrm{cm}$ wide near the pisiform bone. It crossed superficial to the ulnar nerve and artery proximal to the wrist, then accompanied them through Guyon's canal, deep to the volar carpal ligament and palmaris brevis muscle. Distally the accessory head joined the ADM just distal to the pisiform bone. In the hypothenar compartment the ADM had three heads, a lateral head originating from the lateral aspect of the pisiform and pisohamate ligament, an intermediate head originating from the distal end of the pisiform bone, and medial head, with tendinous origin, arising from the carpal bones posterior and distal to the pisiform bone (Fig. 2). Superficial dissection of the forearm just proximal to the wrist joint showed that the tendon of palmaris longus crossed superficial to the median nerve and entered the hand on the 


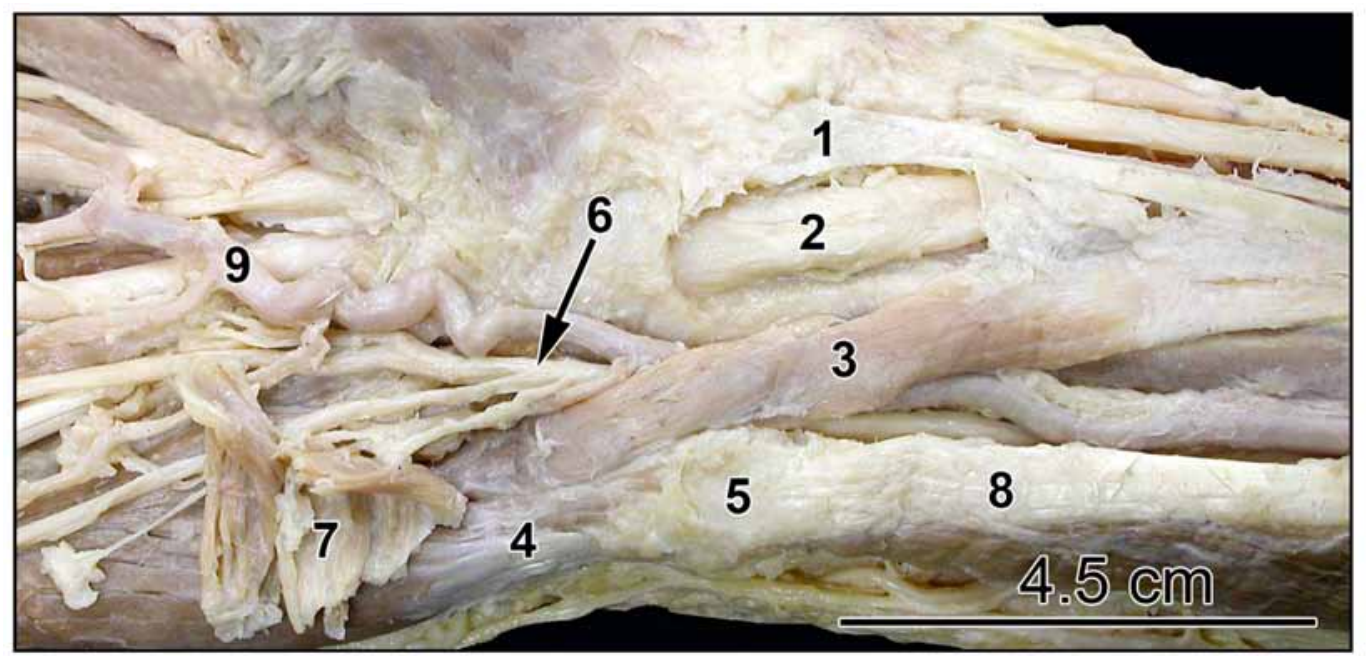

Fig. (1). Superficial dissection of the right hand showing the tendon of palmaris longus (1) located lateral to the median nerve (2). An accessory head $(\mathbf{3})$ of the abductor digiti minim (ADM, 4) has an aponeurotic origin from the medial border of the tendon of the palmaris longus (1). The accessory head is seen crossing the ulnar artery and nerve just above the pisiform bone (5). The superficial division of the ulnar nerve (6) comes to the surface lateral to the accessory head of the ADM and provides innervation to the palmaris brevis (7). Tendon of flexor carpi ulnaris (8); superficial palmar arch (9).

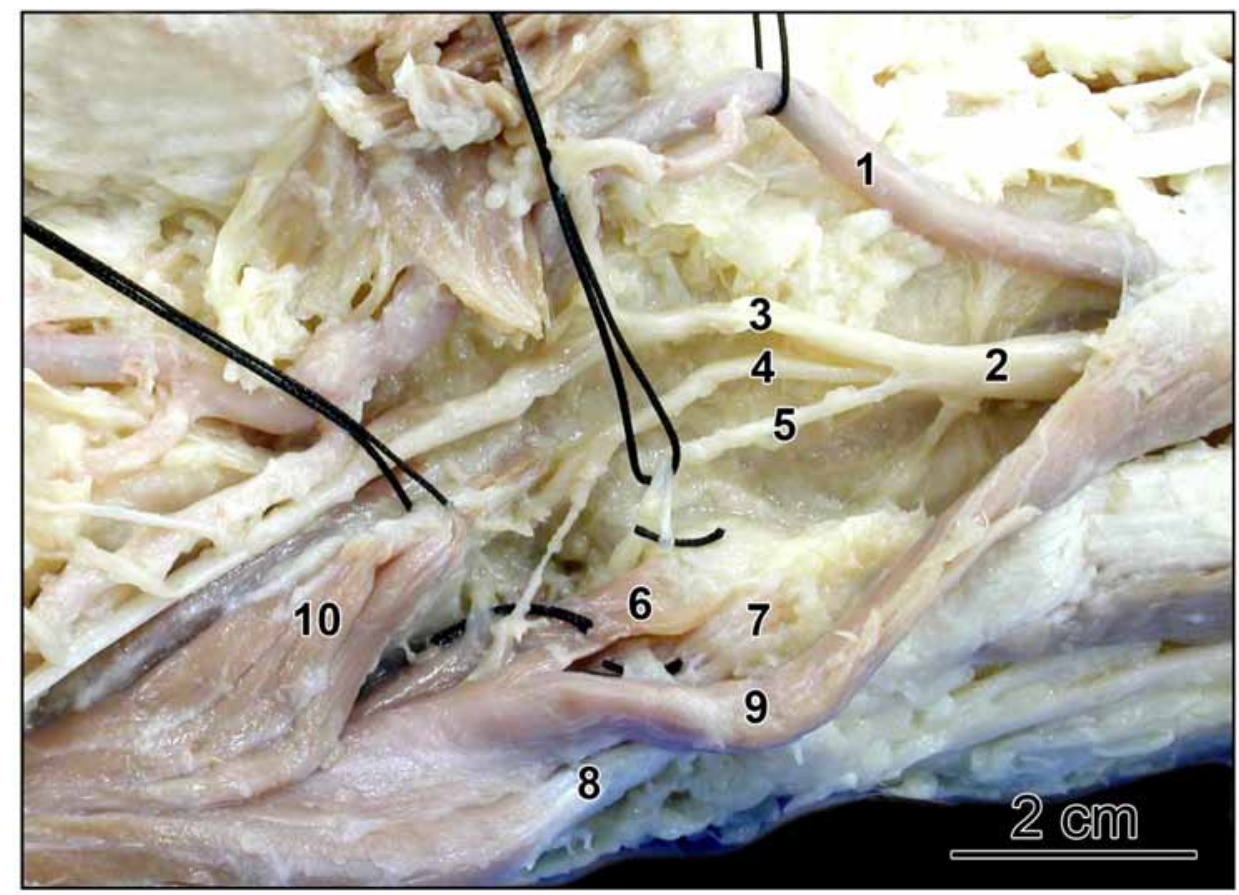

Fig. (2). Deeper dissection of the hand, showing the ulnar artery (1) displaced laterally. Ulnar nerve (2) trifurcation into superficial (3), Deep (4) and muscular (5) branches. The muscular branch (5) provides innervation to the lateral head of ADM (6), then passes deep to it to innervate the intermediate head (7) and medial head (8) of the ADM and the deep surface of the accessory head (9). The deep branch of the ulnar nerve (4) provides a second nerve supply to the lateral head of ADM (6). The branches to the ADM are highlighted by short pieces of silk suture. The flexor digiti minimi (10) is reflected laterally.

medial aspect of the thenar eminence, thus the median nerve appeared on the medial side of the tendon before it entered the carpal tunnel (Fig. 1).

In Guyon's canal the ulnar nerve showed trifurcation into superficial, deep and muscular branches. The muscular branch innervated the lateral head of the ADM then crossed deep to its origin to reach and innervate the intermediate and medial heads and the deep surface of the Accessory ADM (Fig. 2). In addition the deep branch of the ulnar nerve provided a second nerve supply to the lateral head of the ADM.

\section{DISCUSSION}

Variations in branching patterns of nerves and in short muscles of the hand have been seen in clinical practice [4-6] 
and during cadaveric dissections [7, 8]. In the current study an accessory head of the abductor digiti minimi (ADM) was seen in addition to three heads that existed in the hypothenar compartment. This accessory head was seen in 3 out of 120 hands examined in the current study $(2.5 \%)$, while a $22.4 \%$ incidence was reported previously [9]. The accessory head described in the current study closely resembles that described by Soldado-Carrera et al. [10] particularly in the origin of the muscle. Another study reported an accessory head for the ADM, but unlike in the current study, it had a far distal insertion into the base of the proximal phalanx of the little finger and had a round slip of origin from the palmaris longus tendon [11]. In the later study, two other heads were seen in the hypothenar compartment, a superficial and a deep head. Other studies reported a proximal head for the ADM that occupied Guyon's canal but originated from the deep fascia of the lower forearm, and the palmaris longus was absent [12]. Multiple origins for the proximal head of ADM have been reviewed recently [13].

Accessory muscles as described here have been implicated in wrist problems and entrapment neuropathy $[12$, 13] as these often compromise the available space, compressing adjacent vessels and nerves [14]. Knowledge of these anomalies and their predominance are invaluable for diagnosis and selecting the surgical approach for ulnar nerve decompression [14].

A fleshy head arising from the tendon of palmaris longus and joining the hypothenar muscles has been referred to as a variant slip of the palmaris longus. However, on account of its innervation from the ulnar nerve, as seen in the current study, it should be considered a variant of the hypothenar muscles [12].

Typically the tendon of palmaris longus crosses the middle of the wrist joint and becomes continuous with the apex of the palmar aponeurosis. In such case the median nerve appears above the flexor retinaculum in the interval between the palmaris longus and flexor carpi radialis tendons, before passing deep to the flexor retinaculum. In the current study the tendon of palmaris longus inclined more to the radial side entering the hand closer to the thenar eminence with the median nerve appearing on its medial aspect. It is possible that in such situation the accessory belly of the ADM muscle, functionally, acts to adjust the axis of action of the long tendon with reference to the midline of the hand. Such function may be similar to that of the quadratus plantae (flexor accessorius) on the tendon of the flexor digitorum longus in the foot.

The common pattern of ulnar nerve branching is a bifurcation into a superficial, predominantly sensory branch, and a deep motor branch, a pattern seen in 38 out of 50 cases, while trifurcation of the ulnar nerve was seen in 11 cases out of 50 [7]. In the current study the ulnar nerve showed trifurcation in Guyon's canal into superficial, deep and muscular divisions. In the study by Bonnel and Vila [7] the third nerve in the trifurcation was a sensory branch, not a muscular branch as seen in the current study. The trifurcation described in the current study does not resemble any of the five classifications of arborisations described by Murata [8].
Variations in nerve branching are less common than muscle variations. Dissection of 58 hands showed $22.4 \%$ incidence of anomalous muscles but only $1.7 \%$ incidence for anomalous nerves [9]. The trifurcation of the ulnar nerve seen in the current study was symmetrical to that seen in the left hand of the same cadaver [15]. A number of studies have found that anatomical variations often exist bilaterally $[6,9$, 16]. The almost consistent neural branching and innervation of muscles by specific pools of motor neurons in normal development, and the symmetry often seen when neural variations occur, suggest a genetically determined developmental map. Experimental studies in the chick involving cranio-caudal reversal of parts of the neural tube containing several segments showed that labelled axons alter their path and enter the limb plexus in a modified way that allows axons to reach and innervate their predetermined targets $[17,18]$. This indicates that motor neurons actively respond to cues along their path predetermined by an early central specification [18]. Central specialization of motor neurons and their organization along the medio-lateral axis is determined by midline structures, the notochord and floor plate of the neural tube, which express the sonic hedgehog gene with a medial to lateral gradient [19]. Therefore minor variations in gene expression may manifest bilaterally and may explain the symmetric variant of ulnar nerve arborisation seen here. Although trifurcation of the ulnar nerve described here in the right hand was symmetrical to that in the left hand of the same cadaver [15] there was no symmetry in the hypothenar muscles as the accessory head of the ADM was only seen in the right hand.

\section{CONFLICT OF INTEREST}

The authors confirm that this article content has no conflict of interest.

\section{ACKNOWLEDGEMENTS}

The author would like to thank Dr. P.H. Makar.

\section{REFERENCES}

[1] Ozdemir O, Calisaneller T, Gerilmez A, Gulsen S, Altinors N Ulnar nerve entrapment in Guyon's canal due to a lipoma. J Neurosurg Sci 2010; 54: 125-7.

[2] Monacelli G, Rizzo MI, Spagnoli AM, Monarca C, Scuderi N. Ulnar artery thrombosis and nerve entrapment at Guyon's canal: our diagnostic and therapeutic algorithm. In Vivo 2010; 24: 779 82.

[3] Aguiar PH, Bor-Seng-Shu E, Gomes-Pinto F, et al. Surgical management of Guyon's canal syndrome, an ulnar nerve entrapment at the wrist: report of two cases. Arq Neuropsiquiatr 2001; 59: 106-11.

[4] Hankins CL, Flemming S. A variant of Kaplan's accessory branch of the dorsal cutaneous branch of the ulnar nerve: a case report and review of the literature. J Hand Surg Am 2005; 30: 1231-5.

[5] Harvie P, Patel N, Ostlere SJ. Ulnar nerve compression at Guyon's canal by an anomalous abductor digiti minimi muscle: the role of ultrasound in clinical diagnosis. Hand Surg 2003; 8: 271-5.

[6] Zeiss J, Jakab E, Khimji T, Imbriglia J. The ulnar tunnel at the wrist (Guyon's canal): normal MR anatomy and variants. AJR Am J Roentgenol 1992; 158: 1081-5.

[7] Bonnel F, Vila RM. Anatomical study of the ulnar nerve in the hand. J Hand Surg Br 1985; 10: 165-8.

[8] Murata K, Tamai M, Gupta A. Anatomic study of variations of hypothenar muscles and arborization patterns of the ulnar nerve in the hand. J Hand Surg Am 2004; 29: 500-9.

[9] Dodds GA, Hale D, Jackson WT. Incidence of anatomic variants in Guyon's canal. J Hand Surg Am 1990; 15: 352-5. 
[10] Soldado-Carrera F, Vilar-Coromina N, Rodriguez-Baeza A. An accessory belly of the abductor digiti minimi muscle: a case report and embryologic aspects. Surg Radiol Anat 2000; 22: 51-4.

[11] Curry B, Kuz J. A new variation of abductor digiti minimi accessorius. J Hand Surg 2000; 25: 585-7.

[12] Jeffery AK. Compression of the deep palmar branch of the ulnar nerve by an anomalous muscle. Case report and review. J Bone Joint Surg Br 1971; 53: 718-23.

[13] Nayak SR, Jiji PJ. Unusual origin of abductor digiti minimi and its clinical significance. Clin Anat 2007; 20: 972-3.

[14] Ruocco MJ, Walsh JJ, Jackson JP. MR imaging of ulnar nerve entrapment secondary to an anomalous wrist muscle. Skeletal Radiol 1998; 27: 218-21.
[15] Ghabriel MN, Makar PH. Anatomical variations in the ulnar nerve and hypothenar muscles. Int J Anat Var 2011; 4: 131-133.

[16] Netscher D, Cohen V. Ulnar nerve compression at the wrist secondary to anomalous muscles: a patient with a variant of abductor digiti minimi. Ann Plast Surg 1997; 39: 647-51.

[17] Kaplan EB. Variation of the ulnar nerve at the wrist. Bull Hosp Joint Dis 1963; 24: 85-88.

[18] Lance-Jones C, Landmesser L. Pathway selection by chick lumbosacral motoneurons during normal development. Proc R Soc Lond B Biol Sci 1981; 214: 1-18.

[19] Placzek M. The role of the notochord and floor plate in inductive interactions. Curr Opin Genet Dev 1995; 5: 499-506.

(C) Mounir N. Ghabriel; Licensee Bentham Open.

This is an open access article licensed under the terms of the Creative Commons Attribution Non-Commercial License (http://creativecommons.org/licenses/by-nc/3.0/) which permits unrestricted, non-commercial use, distribution and reproduction in any medium, provided the work is properly cited. 